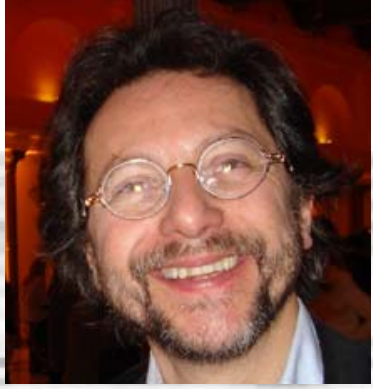

Prof. Dr. Wolfram Koepf Universität Kassel

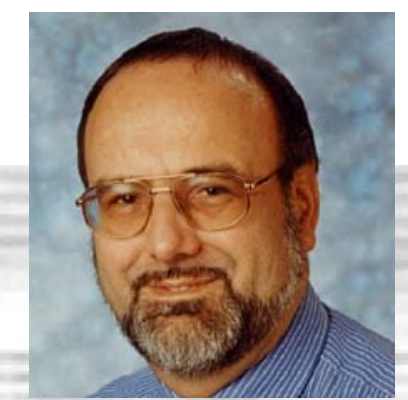

Prof. Dr. Ernst W. Mayr Technische Universität München

\title{
Mathematik und die Informatik
}

Das gerade vergangene Jahr, 2008, war das Jahr der Mathematik. In zahlreichen Veranstaltungen, Ausstellungen und Veröffentlichungen haben es die Organisatoren in beeindruckender Weise vermocht, die grundlegenden wie auch die aktuellen Inhalte der Mathematik einem breiten Publikum in wohl ausgewogener, Interesse erweckender Form nahezubringen. Da die Mathematik ja eine der (gar nicht unwesentlichen) Mütter der Informatik darstellt, berührt das auch uns mit großer Freude und Genugtuung. Nachdem im Jahr 2006, dem Jahr der Informatik, unsere Disziplin die Gelegenheit hatte, sich selbst in ihrer Breite und neuen Durchdringungskraft darzustellen, und nachdem sie dies wohl auch sehr erfolgreich und wirksam bewerkstelligt hat, folgten im Jahr darauf die Geisteswissenschaften, und auch hier zeigte sich die enge Verbundenheit und starke Verzahnung mit der Informatik in sehr, sehr vielen Aspekten, von der Grundlagenforschung bis hin zu den ganz aktuellen Themen.

2008 war dann, mit der Mathematik, wieder eine der ganz wesentlichen Grundlagen der Informatik an der Reihe. Denn neben den Anwendungen, die wir heute in sehr starkem Maße und in fast allen Bereichen der modernen Wissenschaft und Gesellschaft beobachten können, und neben den technischen und technologischen Grundlagen im Bereich der Material-, der Elektronik- und Nachrichten- und der Mikrotechnologie, bildet die Mathematik eben eine ganz wesentliche Wurzel der Informatik. Sie hat ihr, angefangen mit zahlreichen formalen Fundamenten, aber auch mit sehr weitgreifenden und tiefen Theorien und Kalkülen, ein sehr fest gefügtes und trotz aller immensen, heute beobachtbaren Dynamik sehr robustes und dauerhaftes Fundament verliehen. Von diesem Fundament, das große Baublöcke z.B. in der Logik, in der Algebra, der Kombinatorik, der Rekursionstheorie, aber auch in Bereichen wie der Zahlentheorie und der Numerik hat, hat es dann die Informatik im Lauf der Zeit geschafft, immer wieder neue, interessante, durch von Problemen aus Anwendungsfeldern motivierte neue, erweiterte Theorien und Werkzeuge hinzuzufügen sowie ein ganz großes und immens wichtiges Arbeitsgebiet zu erstellen, das sich mit der Schaffung und Handhabung großer komplexer Systeme beschäftigt, wie sie ja zahlreiche der heute verfügbaren großen Softwaresysteme par excellence darstellen.

Im diesem Heft des Informatik-Spektrums möchten wir einige Beispiele für die sehr enge und sehr natürliche Verbindung, ja Symbiose, zwischen der Mathematik und der Informatik aufzeigen, die aus dem Bereich der Computeralgebra stammen, genau dem Gebiet also, in dem die typisch mathematische Algebra sich der leistungsfähigen Methoden und Technologien der Informatik bedient und so erstaunliche Fortschritte zu erzielen vermag.

Der Beitrag "Alles logisch oder was?" von Kreuzer und Kühling berührt ein Gebiet, das sehr eng mit der Informatik verwandt ist, die mathematische Logik. Der Artikel zeigt, wie beim korrekten logischen Schlussfolgern effiziente Methoden der Computeralgebra eingesetzt werden können.

Im Artikel „Integralrechnung und Computeralgebra" zeigt Koepf die historische Entwicklung der Integralrechnung von der Antike bis hin zu modernen algorithmischen Methoden auf. Seit es den Risch-Algorithmus gibt, spielen heuristische Methoden hier eine weit geringere Rolle.

Kreuzer und Poulisse stellen in "Algebraisches Erdöl" genauso wie Laubenbacher und Sturmfels in "Computeralgebra in der Systembiologie" zwei konkrete Industrieanwendungen der Computeralgebra vor, an die man zunächst 


\section{$\{$ EDITORIAL}

wohl nicht unmittelbar denken mag, die aber die inhärente Breite und das Potential des Gebiets beleuchten. Es ist zu betonen, dass erst durch die Leistungsfähigkeit heutiger moderner Computer derartige Anwendungen möglich werden.

Zwei hochinteressante aktuelle Systeme, "GeoGebra" von Hohenwarter und "FeliX" von Oldenburg, die dynamische Geometrie und algebraische Methoden unter einem Dach vereinigen und die noch nicht so bekannt sind, werden von den Autoren selbst nun einem breiteren Publikum vorgestellt.

Schließlich zeigt Günter Ziegler in seinem Beitrag "Primzahltests und Primzahlrekorde", auf welch breites Interesse spezielle Fragestellungen der Zahlentheorie, die auch in der modernen Kryptographie bedeutsam sind, stoßen. Auf den hier angesprochenen mathematischen Konzepten und Modellen beruhen sehr viele der modernen kryptographischen Systeme, wie sie z.B. bei Bank- und Kreditkarten zur Verwendung kommen.

Kassel und München, im Januar 2009

Wolfram Koepf und Ernst W. Mayr 